\title{
Relationship between myocardial edema and left ventricular wall thickness in acute myocardial ischemia: A Magnetic Resonance I maging study
}

\author{
Yoko Mikami ${ }^{1}$, Andreas Kumar ${ }^{2}$, Hassan Abdel-Aty ${ }^{3}$, and Matthias G. Friedrich ${ }^{1,4}$ \\ 1. Stephenson CMR Centre at the Libin Cardiovascular Institute of Alberta, Calgary, Canada. 2. University of Toronto, St. \\ Michael's Hospital, Toronto, Ontario, Canada. 3. Spital Netz Bern, Morillonstrasse, Bern, Switzerland. 4. Montreal Heart \\ Institute, Rue Bélanger, Montréal, Québec, Canada \\ Correspondence: Yoko Mikami. Address: Stephenson CMR Centre at the Libin Cardiovascular Institute of Alberta Suite \\ 0700, SSB, 1403-29th Street NW, Calgary, Canada. Email: ymikami@ucalgary.ca
}

Received: January 27, $2014 \quad$ Accepted: February 26, $2014 \quad$ Online Published: March 20, 2014

DOI : 10.5430/ijdi.v1n2p23 URL: http://dx.doi.org/10.5430/ijdi.v1n2p23

\section{Abstract}

Purpose: We sought to assess the relationship between left ventricular regional end-diastolic myocardial wall thickness (EDWT) and myocardial edema defined using T2-weighted Cardiovascular Magnetic Resonance (CMR) after acute myocardial ischemia and reperfusion.

Methods: T2-weighted and cine CMR images for $7 \mathrm{dogs}$ at baseline, during coronary occlusion (mean $33 \pm 4$ minutes) and after reperfusion were studied. The EDWT was measured in segments with high signal intensity (SI) on T2-weighted images, adjacent segments and remote segments according to a 16-segment model.

Results: The EDWT after reperfusion in segments with high SI on T2-weighted images was significantly increased compared to baseline $(6.28 \pm 1.06 \mathrm{~mm}$ and $5.51 \pm 1.40 \mathrm{~mm}, \mathrm{p}<0.05)$, whereas EDWT after the reperfusion in adjacent and remote segments did not show significant difference compared to baseline (adjacent: $6.48 \pm 1.55 \mathrm{~mm}$ and $6.38 \pm 1.26 \mathrm{~mm}$, $\mathrm{p}=$ N.S., remote: $6.41 \pm 1.11 \mathrm{~mm}$ and $6.42 \pm 1.27 \mathrm{~mm}, \mathrm{p}=\mathrm{N} . \mathrm{S}$.). The $\%$ increase in EDWT after reperfusion from baseline in segments with high SI on T2-weighted images was higher than those in adjacent and remote segments $(19 \pm 30 \%, 1.3 \pm$ $15 \%$ and $1.5 \pm 16 \%$, respectively, $\mathrm{p}<0.05$ ).

Conclusions: After a brief period of ischemia and reperfusion, edema as defined by high SI on T2-weighted CMR is related to an increase in EDWT. This increase however is too small to be clinically relevant to be used for the detection of acute myocardial injury. Edema imaging is more sensitive and is an essential part of the reliable assessment of acute ischemic myocardial injury.

\section{Keywords}

Myocardial edema, T2-weighted cardiovascular magnetic resonance, Myocardial ischemia, Left ventricular wall thickness

\section{I ntroduction}

The early detection of acute ischemic injury and restoration of coronary blood flow have beneficial effects on patient outcomes. T2-weighted cardiovascular magnetic resonance (CMR) imaging sensitively detects ischemia-related 
myocardial edema ${ }^{[1-5]}$, an early marker of acute myocardial injury. A recent study has shown that T2-weighted CMR imaging of edema detects acute ischemic injury before the onset of irreversible injury ${ }^{[6]}$.

Experimental studies using echocardiography have shown that reperfusion of acutely infarcted segments is associated with an increase of myocardial end-diastolic wall thickness (EDWT) ${ }^{[7-10]}$. In a study with pigs subjected to 90 minutes of ischemia followed by 60 minutes of reperfusion, Turschner et al. reported that wall thickness changes after reperfusion were due to histological features of massive extracellular edema ${ }^{[9]}$. Smulders et al. recently reported the use of increased EDWT measured on CMR to determine the infarct age with patients with acute myocardial infarction ${ }^{[11]}$. In their study, the combination of increased EDWT or the presence of microvascular obstruction showed higher accuracy in differentiating between acute and old myocardial infarctions compared to T2-weighted CMR alone. However, the change in wall thickness at a very early stage of ischemic injury, especially in relation to tissue edema, has not been investigated.

We investigated the relationship between myocardial edema as identified on T2-weighted CMR after brief episodes of ischemia and increase in regional EDWT.

\section{Materials and methods}

Fifteen mongrel dogs (weight 15 to $25 \mathrm{~kg}$ ) were studied in accordance with the Position of the American Heart Association on Research Animal Use and with local ethics committee approval. Data from these animals have been published in a previous study ${ }^{[6]}$ reporting on the presence of myocardial edema early during ischemia, before the onset of irreversible injury.

\subsection{Surgical preparation}

Anesthesia was induced with $30-\mathrm{mg} / \mathrm{kg}$ sodium thiopental and maintained using $4 \mathrm{mg} / \mathrm{h}$ fentanyl citrate and $25 \mathrm{mg} / \mathrm{h}$ midazolam. Animals were intubated and respirated using Harvard constant volume ventilation with an $\mathrm{O}_{2}$ and $\mathrm{NO}_{2}$ gas mixture. The chest wall was opened with left lateral thoracotomy. Lidocaine was administered $(1 \mathrm{mg} / \mathrm{kg}, 3$ times $5 \mathrm{~min}$ apart, and then a constant drip $1 \mathrm{mg} / \mathrm{min}$ ). A snare was placed around the left anterior descending (LAD) artery below the first diagonal artery.

Volume data and wall thickness data for occlusion and reperfusion were available for 7 dogs.

\subsection{Protocol for MRI}

The CMR studies were performed using a 1.5 T system (Avanto, Siemens Medical Solutions, Erlangen, Germany). All sequences (T2, Steady-State Free-Precession, and late gadolinium enhancement) were acquired with a slice thickness of $10 \mathrm{~mm}$ with no gap in identical slice positions.

\subsubsection{Baseline}

After acquisition of initial localizer images, retrospectively gated Steady-State Free-Precession (SSFP) images were acquired in short-axis views, encompassing the entire left ventricle (effective repetition time: $40 \mathrm{~ms}$, echo time: $1.1 \mathrm{~ms}, 25$ phases). Breath-hold triple inversion recovery fast spin echo T2-weighted images were then acquired in the same slice locations and planes as the SSFP images (repetition time: $2 \mathrm{R}-\mathrm{R}$ intervals, echo time: $61 \mathrm{~ms}$, field of view: 34 to $38 \mathrm{~cm}$, matrix: $256 \times 256$, echo train length: 15, acquisition window: $150 \mathrm{~ms}$, inversion time: $170 \mathrm{~ms}$ ). For T2-weighted imaging, we acquired images using an 8-channel surface coil with a signal intensity gradient correction algorithm (dogs \#1-\#7), or a body coil (dogs \#8-\#15) ${ }^{[6]}$. Within the 7 dogs analyzed for T2-weighted images, a surface coil was used for 2 dogs and a body coil for 5 dogs. The signal intensity gradient correction was applied as follows: a pre-scan normalization filter with a low resolution, large field of view scan was acquired. The filter was then smoothed to remove spikes originating from occasional non-Gaussian nature of the image noise. Scanned data with the surface coil were weighted using the coil characteristic/pre-scan information to remove inhomogeneities from the surface coil. 


\subsubsection{Occlusion}

Immediately after LAD occlusion, SSFP and T2-weighted images were acquired. We obtained T2-weighted images every 4 to 5 min, until a focal high T2 signal intensity in LAD area in at least 2 consecutive short-axis slices was visually detectable by 2 agreeing observers.

\subsubsection{Reperfusion}

Once a focal high T2 signal was detected, the coronary artery occlusion was removed. T2-weighted images and SSFP images were acquired right after the coronary occlusion was removed. Then, $0.2 \mathrm{mmol} / \mathrm{kg}$ body weight of gadolinium-diethylenetriamine pentaacetic acid (Magnevist ${ }^{\circledR}$, Bayer Healthcare, Toronto, Ontario, Canada) was intravenously injected and late gadolinium enhanced (LGE) images with an inversion time selected to minimize signal of normal myocardium (TI individually determined, range 200 to $300 \mathrm{~ms}$ ) were acquired 10 minutes after injection of the contrast agent.

\subsection{I mage analysis}

We used certified software for the evaluation ( $\mathrm{cvi}^{42}$, Circle CVI, Calgary, AB, Canada). The analysis was performed using a 16-segment model based as proposed by the AHA, dividing the left ventricular (LV) myocardium into six basal, six mid-ventricular, and four apical segments ${ }^{[12]}$. The SSFP images for baseline, during ischemia and after reperfusion were analyzed by the consensus of two readers (Y.M., A.K.). Endocardial and epicardial contours were manually drawn in diastole and systole for every short-axis slice with exclusion of the papillary muscles (see Figure 1A). We measured and calculated the following parameters: end-diastolic volume (EDV), end-systolic volume (ESV), ejection fraction (EF), and LV myocardial mass (LVmass). End-diastolic wall thickness was also calculated automatically for each segment. The analysis of the T2-weighted images was performed on a separate day from the SSFP analysis in a random order blinded to the results of EDWT. Presence or absence of edema on T2-weighted images acquired after reperfusion was determined on each segment. The segment was regarded as edematous when the myocardium revealed a signal intensity (SI) of more than 2SD above the mean SI of remote myocardium (see Figure 1B).
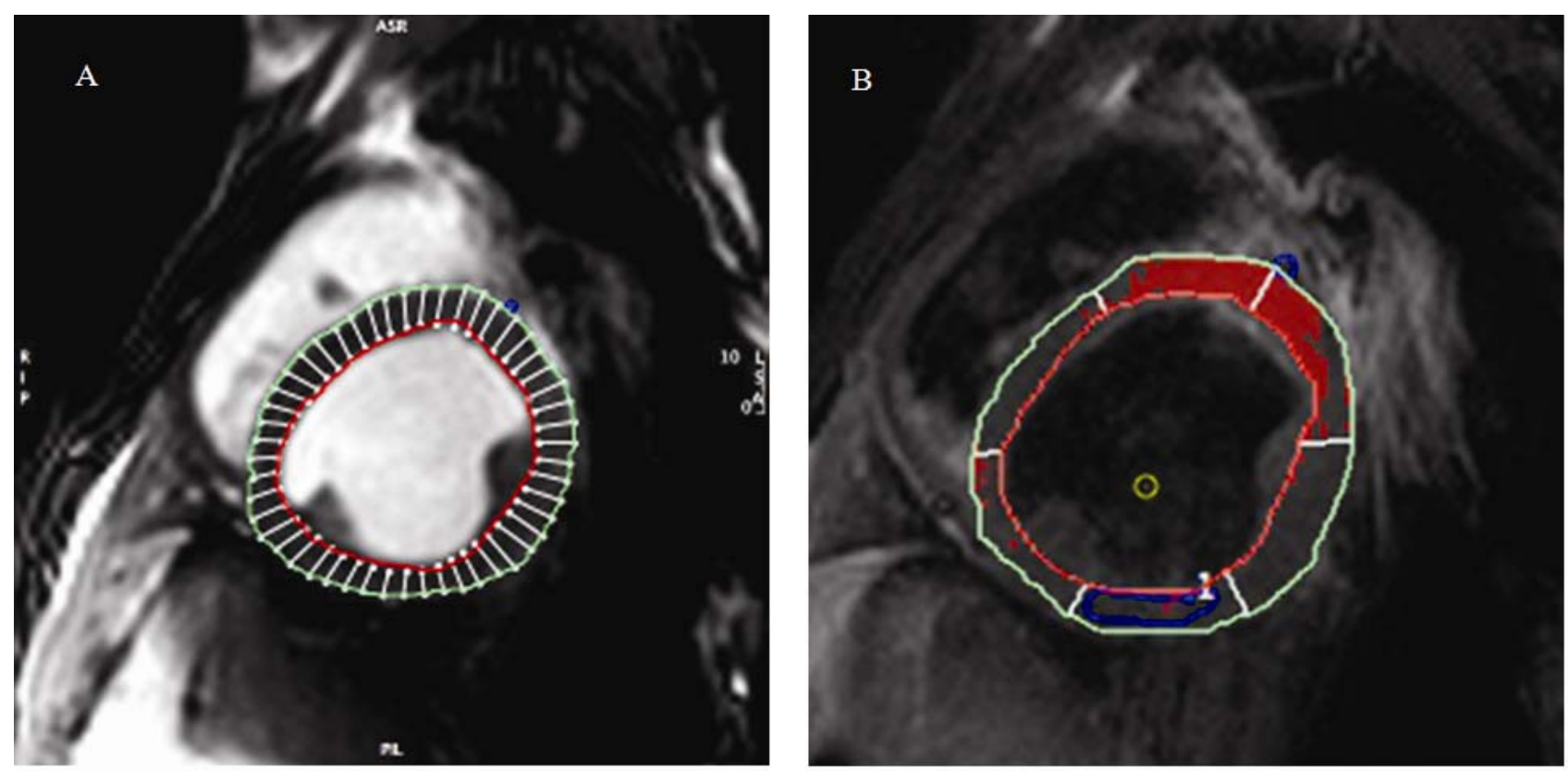

Figure 1. (A) Measurement of end-diastolic wall thickness (EDWT) (Left). (B)Semi-quantitative assessment of presence of edema on T2-weighted CMR using 2SD above the mean of normal myocardium as a threshold (Right). High signal intensity segments are observed in the antero-septal wall. 


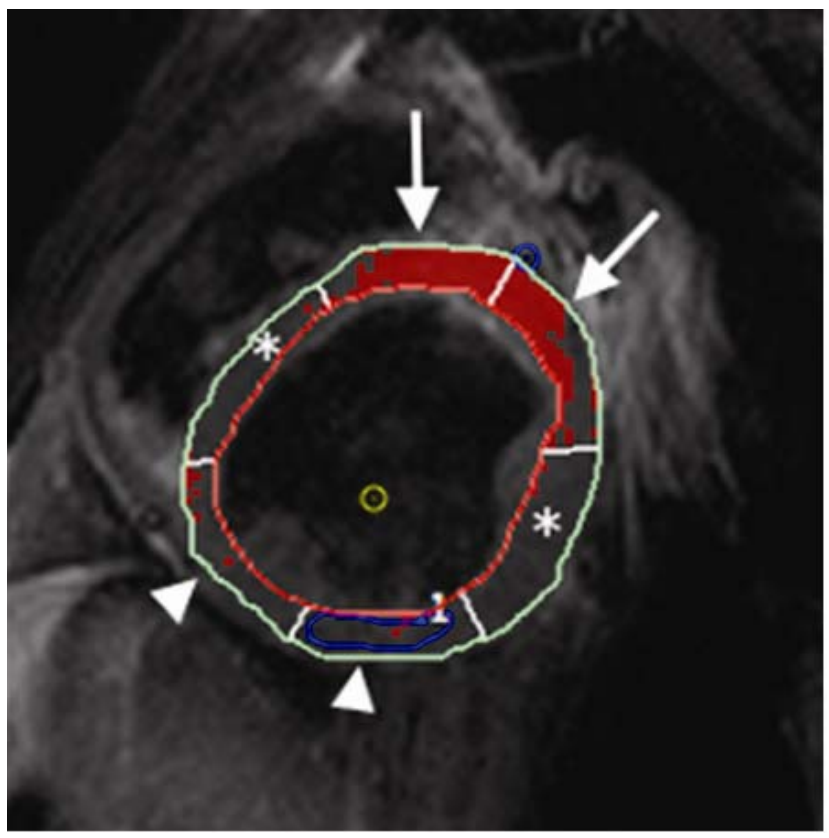

Figure 2. Segments with high signal intensity (arrows), segments adjacent to segments with high signal intensity $(*)$, and remote segments (arrow heads) on T2-weighted images.

The presence or absence of infarction was evaluated on LGE images using the SI of mean + 5SD of remote myocardium as a threshold ${ }^{[13]}$. After the image analysis, the values from SSFP, T2-weighted images and LGE were matched by slice location. Values for EDWT were compared between baseline, during occlusion of the LAD and after reperfusion in segments with semi-quantitatively identified high SI on T2-weighted images, segments adjacent to segments with high SI on T2-weighted images, and remote segments (see Figure 2). The \% increase in EDWT from baseline was also calculated in each of these segments by the following formula: (EDWT reperfusion - EDWT baseline)/ EDWT baseline $\times 100$. The EDWT measurement was repeated on 15 randomly selected slices to test the reproducibility.

\subsection{Statistical analysis}

All values are presented as mean \pm SD. Statistical analysis was performed using commercially available software (SPSS 19.0 for Macintosh, SPSS, Chicago, Illinois). The EDWT between baseline, during occlusion and after reperfusion for each segment were compared by one-way repeated measures ANOVA. Pairwise comparison with Bonferroni procedure was performed as post-hoc analysis. Also the LV volume, global LV function and LV mass at baseline, occlusion and after reperfusion were compared by one-way repeated measures ANOVA with pairwise comparison with Bonferroni procedure as post-hoc test. The \% increase in EDWT from baseline among each segments were compared by one-way ANOVA, with the Student-Newman-Keuls test as post-hoc analysis. A $p$ value of $<0.05$ was considered to be significant.

\section{Results}

In $1 \mathrm{dog}$, the study had to be interrupted due to technical problems. Five dogs died either before occlusion $(\mathrm{n}=1)$, immediately after occlusion $(n=1)$, during occlusion $(n=1)$, or after reperfusion $(n=2)$. One dog was excluded from this study since the period of ischemia was much longer (about 90 minutes) compared to other dogs. Short-axis SSFP images were not acquired for $1 \mathrm{dog}$ after reperfusion for technical reasons. Thus, 92 segments from 7 dogs were used for the segment-based analysis of EDWT ( 1 slice was excluded in 2 dogs due to insufficient cine image quality and 2 slices were excluded in 1 dog due to insufficient T2-weighted image quality). One dog had ventricular fibrillation right before 
reperfusion and was scanned after successful defibrillation. The mean duration of ischemia for the 7 dogs analyzed was $33 \pm 4 \min (27$ to $41 \mathrm{~min})$.

Table 1 shows the mean change in LV volume, function, and mass during the experimental protocol. Results are shown for 7 dogs. There was no LV regional wall motion abnormality at baseline. Compared with baseline, we observed an increase of end-diastolic volume during occlusion, and an increase of end-systolic volumes with a decrease of LV ejection fraction during occlusion and after reperfusion.

Bland-Altman plots for repeated EDWT measurement is shown in Figure 3.

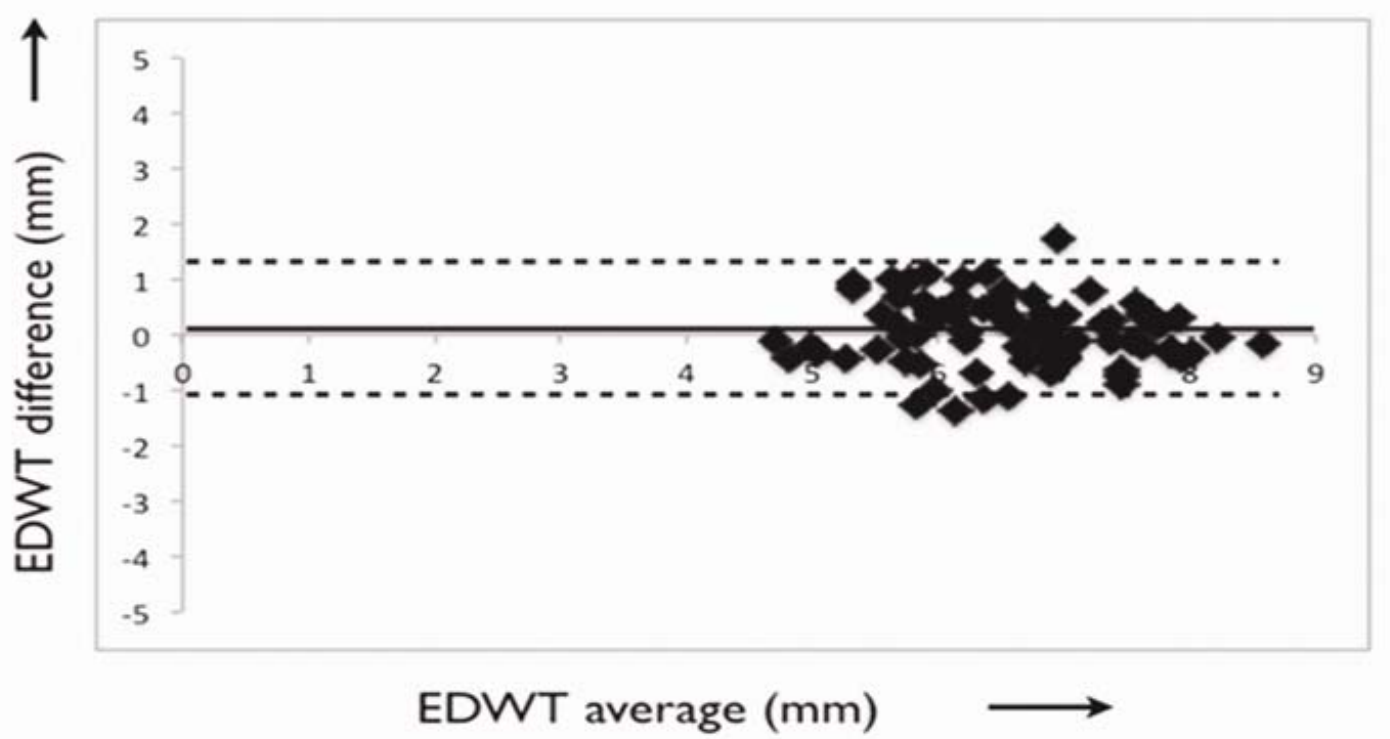

Figure 3. Bland-Altman plots for the repeated measurement of End Diastolic Wall Thickness (EDWT). The mean difference and $95 \%$ limits of agreement was $0.06 \pm 1.17$.

Table 1. Changes of LV volume and global function $(\mathrm{N}=7)$

\begin{tabular}{cccc}
\hline & Baseline & Occlusion & Reperfusion \\
\hline EDV (ml) & $75 \pm 13$ & $86 \pm 13^{*}$ & $80 \pm 10$ \\
ESV (ml) & $46 \pm 10$ & $63 \pm 12^{*}$ & $58 \pm 8^{*}$ \\
EF (\%) & $39 \pm 8$ & $27 \pm 5^{*}$ & $28 \pm 5^{*}$ \\
LV mass (g) & $48 \pm 5$ & $50 \pm 4$ & $48 \pm 2$ \\
\hline
\end{tabular}

Note. $\mathrm{EDV}=$ end diastolic volume; $\mathrm{ESV}=$ end systolic volume; $\mathrm{EF}=$ ejection fraction. ${ }^{*} p<0.05$ vs. baseline

EDWT in lateral segments (AHA segment 5, 6, 11, 12, 16) at baseline was more than in anterior-anteroseptal (AHA segments $1,2,7,8,13,14)$ and inferior-inferoseptal (AHA segments $3,4,9,10,15)$ segments $(6.92 \pm 1.07 \mathrm{~mm}$ and $5.64 \pm$ $1.36 \mathrm{~mm}, 5.90 \pm 1.29 \mathrm{~mm}$, respectively, $p<0.05)$.

Twenty-nine segments showed high SI on T2-weighted images, 30 segments were defined as adjacent segments and 33 segments were defined as remote segments. Two segments out of 29 segments with high SI showed infarction on LGE images. Table 2 shows baseline EDWT, EDWT during occlusion and EDWT after reperfusion for each segment. There was a statistically significant intervention effect on EDWT in segments with high SI on T2-weighted images 
$(\mathrm{F}(2,56)=12.958, p<0.001)$ and adjacent segments $(\mathrm{F}(2,58)=7.576, p<0.01)$, whereas there is no such effects in remote segments $(\mathrm{F}(2,64)=2.156, p=\mathrm{N}$. S). Compared to baseline, the EDWT after the reperfusion in segments with high SI on T2-weighted images was significantly increased $(6.28 \pm 1.06 \mathrm{~mm}$ and $5.51 \pm 1.40 \mathrm{~mm}, p<0.05)$, whereas EDWT after the reperfusion in adjacent segments and remote segments did not show a significant change (adjacent segments: $6.48 \pm 1.55 \mathrm{~mm}$ and $6.38 \pm 1.26 \mathrm{~mm}, p=$ N.S., remote segments: $6.41 \pm 1.11 \mathrm{~mm}$ and $6.42 \pm 1.27 \mathrm{~mm}, p=$ N.S.). The EDWT in adjacent segments during occlusion $(5.84 \pm 1.20 \mathrm{~mm})$ was significantly less compared to baseline $(6.38 \pm$ $1.26 \mathrm{~mm}, p<0.05)$ and after reperfusion $(6.48 \pm 1.55 \mathrm{~mm}, p<0.01)$.

Table 2. EDWT at baseline, during LAD occlusion and after reperfusion

\begin{tabular}{cccc}
\hline & & EDWT (mm) \\
\hline Segments with high SI on T2W images $(\mathrm{n}=29)$ & Baseline & Occlusion & Reperfusion \\
Segments without high SI on T2W images & $5.51 \pm 1.40$ & $5.04 \pm 1.01$ & \\
Adjacent segments $(\mathrm{n}=30)$ & & & \\
Remote segments $(\mathrm{n}=33)$ & $6.38 \pm 1.28$ & $5.84 \pm 1.20^{*}$ & $6.48 \pm 1.55$ \\
\hline
\end{tabular}

Note $* p<0.05$ vs. baseline EDWT $=$ end diastolic wall thickness; LAD = left anterior descending artery; $\mathrm{SI}=$ signal intensity; $2 \mathrm{~W}$ images $=$ T2-weighted images.

Figure 4 shows the mean \% increase in EDWT after reperfusion. The \% increase in segments with high SI on T2-weighted images was significantly higher than those in adjacent segments and remote segments $(19 \pm 30,1.3 \pm 15$ and $1.5 \pm 16$, respectively). The $\%$ increase in adjacent segments was not significantly different compared to remote segments $(1.3 \pm 15$ and $1.5 \pm 16, p=$ N.S.). No apparent change in wall thickness was observed visually (see Figure 5).

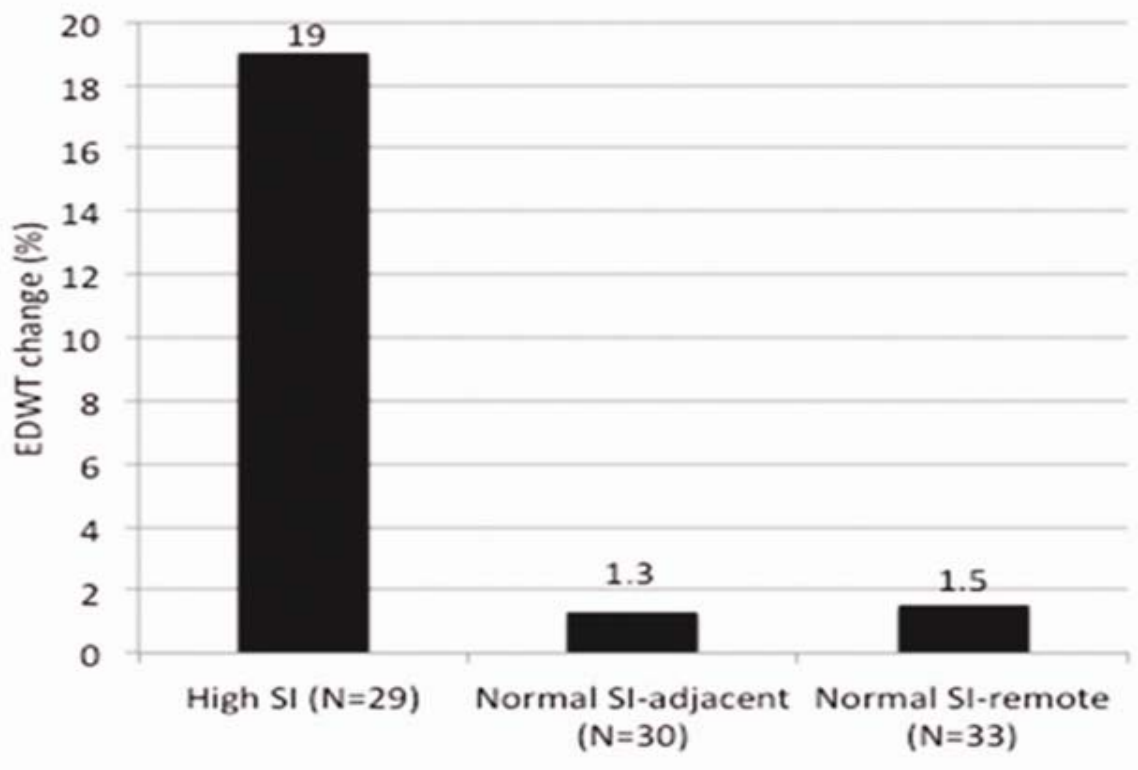

EDWT=end diastolic wall thickness; $\mathrm{SI}=$ signal intensity.

Figure 4. Mean \% change of EDWT by reperfusion. The \% increase in segments with high signal intensity on T2-weighted images was significantly higher than those in adjacent segments and remote segments. The $\%$ increase in adjacent segments did not show significant difference compared to remote segments. 

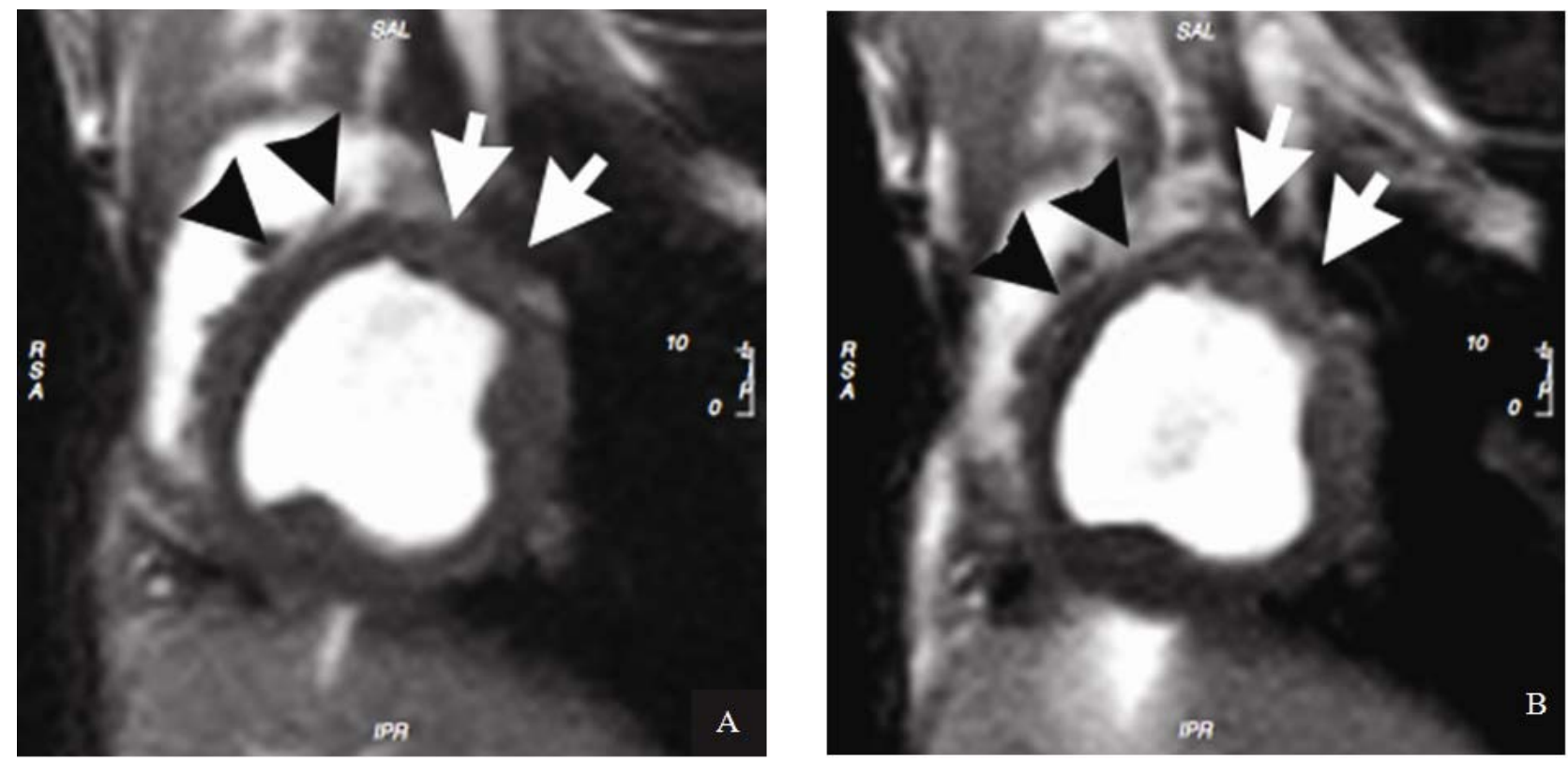

Figure 5. Short axis images at baseline (A, Left) and after reperfusion (B, Right). The wall thickness in the anterior segment (arrows) increased by $31 \%$ and the anterior septum (arrowheads) increased 32\% between baseline and reperfusion. However, the increase is not visually apparent.

\section{Discussion}

Our study indicates that after ischemia with subsequent reperfusion, myocardial edema as assessed by T2-weighted CMR is associated with an increase in regional end-diastolic wall thickness. To the best of our knowledge, this is the first report that shows a direct relationship between myocardial injury visualized by an in vivo imaging technique at a very early stage of ischemic injury and an increase in wall thickness. However, the absolute increase in EDWT after a mean of 33 minutes ischemia and reperfusion from baseline was less than $1 \mathrm{~mm}$, which is too small to be of clinical value to be used for the detection of acute myocardial injury.

The increase in wall thickness in ischemic area in our study was smaller compared to the previous studies using echocardiography in pig models, which showed $98 \pm 28 \%$ increase in EDWT after 90 minutes of ischemia and 5 minutes of reperfusion ${ }^{[10]}$, or $102 \pm 12 \%$ increase after 100-150 minutes of ischemia and 30-60 minutes of reperfusion ${ }^{[8]}$. This could be due to the difference in duration of ischemia between those studies (90-150 minutes) and our study (33 minutes). One possible reason would be that the amount of tissue edema (tissue water content) might have been smaller in our study, resulting in a smaller increase in wall thickness. In experimental animal studies, brief episodes of ischemia (15 minutes) and reperfusion ( 2 minutes) showed no significant change in tissue water ${ }^{[14]}$. On the other hand, studies with longer ischemic periods ( 2 hours by Karolle et al. ${ }^{[15]}$ and 75 min by Klein et al. ${ }^{[16]}$ ) showed a significant increase in water content after reperfusion. The increase in myocardial water content after a long period of ischemia may be due to the capillary membrane damage and leakage of water from the intravascular space to the interstitial space. Kloner et al. showed in an animal study with 20,40,60, 90 or 180 minutes of ischemia that microvascular damage became prominent after 60-90 minutes of coronary occlusion ${ }^{[17]}$. Although tissue water content was not measured in any of the previous studies on echo wall thickness changes, it is tempting to speculate that after 90 minutes of ischemia in previous studies, microvascular damage and greater interstitial edema lead to an increase in wall thickness greater than our study. Our previous study using the same dogs as the present study (33 minutes of ischemia) showed that myocardial water content in ischemic segments was only $1.8 \%$ greater than remote segments ${ }^{[6]}$. 
T2-weighted CMR sensitively detects acute myocardial injury even after a very short period of ischemia where myocardial necrosis has not taken place ${ }^{[6]}$. The absolute water content is not the only mechanism causing T2 changes in ischemia. In fact, a previous study showed that T2 lengthening in the myocardium during ischemia was associated with only a $3 \%$ increase of overall water content ${ }^{[18]}$. Other mechanisms such as a shift of water from the extracellular to the intracellular compartment ${ }^{[19]}$, and the release of bound water molecules from proteins ${ }^{[20,21]}$ due to ischemia-induced acidosis ${ }^{[22]}$ have been shown to have more impact on T2 signal change. Experimental data have shown that this intracellular water fractionation has a stronger impact on T2 than the overall water content ${ }^{[23]}$. Thus, in our study with a short period of ischemia, T2-weighted CMR detected reversible myocardial injury even before a significant increase in absolute water content took place. Recent technical advancements in diffusion weighted images (DWI) for detecting acute myocardial injury ${ }^{[24,25]}$ may contribute to further investigations in the relationship between the state of the water within the myocardium and corresponding signal changes.

Another possible reason for the observed difference would be more severe myocardial relaxation impairment after a long period of ischemia. Increased LV diastolic pressure was observed in studies with human angina pectoris models ${ }^{[26,27]}$ and animal models ${ }^{[28]}$ that result in impaired LV relaxation. The impaired LV relaxation may have been a contributing factor to the increase in EDWT values. A study in the canine model with different ischemic duration showed that the increase in diastolic chamber stiffness is only observed after more than 60 minutes of ischemia ${ }^{[29]}$.

There was a difference in wall thickness between lateral segments and the anterior-anteroseptal and inferior-inferoseptal segments at baseline. However, this would not be a confounding factor as we compared wall thickness of the exact same segments at baseline, during occlusion, and after reperfusion with pairwise comparison.

Some potential limitations should be acknowledged in the present study. The number of the dogs used was limited. We have not performed a histological analysis of the ischemic segments to confirm our speculation that the increase in T2 signal takes place without a significant increase in absolute water content. However, a strong body of evidence supports that T2-weighted signal intensity changes reflect the changes in the molecular environment and/or the quantity of intracellular water ${ }^{[23,30-32]}$. Furthermore, histological analysis of edema is extremely difficult and thus unreliable ${ }^{[33]}$.

\section{Conclusion}

In conclusion, after a brief period of ischemia and reperfusion, edema as defined by high SI on T2-weighted CMR is related to an increase in myocardial EDWT. However, the increase in EDWT is too small to be clinically relevant. Edema imaging is more sensitive and is an essential part of the reliable assessment of acute ischemic myocardial injury.

\section{Acknowledgments}

The authors would like to thank Myra Cocker, Ph.D; Jaqueline Flewitt, MSc; Loreen Thon, RT; and Cheryl Meek, RN for the excellent assistance and John Tyberg, MD, Ph.D for his valuable expertise and very helpful discussions. The authors also thank Tak Fung, Ph.D for his statistical assistance.

\section{Competing interests}

MGF is a board member of Circle Cardiovascular Imaging Inc, Calgary, the manufacturer of the software used for the evaluation. The other authors have no conflicts of interest.

\section{References}

[1] Brown JJ, Peterson TM, Slutsky RA. Regional myocardial blood flow, edema formation, and magnetic relaxation times during acute myocardial ischemia in the canine. Invest Radiol. 1985 Aug; 20(5): 465-71. 
[2] Johnston DL, Homma S, Liu P, et al. Serial changes in nuclear magnetic resonance relaxation times after myocardial infarction in the rabbit: relationship to water content, severity of ischemia, and histopathology over a six-month period. Magn Reson Med. 1988 Dec; 8(4): 363-79.

[3] Bouchard A, Reeves RC, Cranney G, Bishop SP, Pohost GM. Assessment of myocardial infarct size by means of T2-weighted 1H nuclear magnetic resonance imaging. Am Heart J. 1989 Feb; 117(2): 281-9.

[4] Choi SI, Jiang CZ, Lim KH, et al. Application of breath-hold T2-weighted, first-pass perfusion and gadolinium-enhanced T1-weighted MR imaging for assessment of myocardial viability in a pig model. J Magn Reson Imaging. 2000 May; 11(5): $476-80$.

[5] Dymarkowski S, Ni Y, Miao Y, et al. Value of t2-weighted magnetic resonance imaging early after myocardial infarction in dogs: comparison with bis-gadolinium-mesoporphyrin enhanced T1-weighted magnetic resonance imaging and functional data from cine magnetic resonance imaging. Invest Radiol. 2002 Feb; 37(2): 77-85.

[6] Abdel-Aty H, Cocker M, Meek C, et al. Edema as a very early marker for acute myocardial ischemia: a cardiovascular magnetic resonance study. J.Am.Coll.Cardiol. 2009 Apr 7; 53(14): 1194-201.

[7] Gaasch WH, Bernard SA. The effect of acute changes in coronary blood flow on left ventricular end-diastolic wall thickness. An echocardiographic study. Circulation. 1977 Oct; 56(4 Pt 1): 593-8.

[8] Pislaru C, Bruce CJ, Seward JB, et al. Distinctive changes in end-diastolic wall thickness and postsystolic thickening in viable and infarcted myocardium. J Am Soc Echocardiogr. 2004 Aug; 17(8): 855-62.

[9] Turschner O, D'Hooge J, Dommke C, et al. The sequential changes in myocardial thickness and thickening which occur during acute transmural infarction, infarct reperfusion and the resultant expression of reperfusion injury. Eur.Heart J. 2004 May; 25(9): 794-803.

[10] Streb W, Marciniak M, Claus P, et al. Full or pressure limited reperfusion of an acute myocardial infarct results in a different wall thickness and deformation of the distal myocardium--implications for clinical reperfusion strategies. Eur J Echocardiogr. 2008 Jul; 9(4): 458-65.

[11] Smulders MW, Bekkers SC, Kim HW, et al. A simple visual algorithm incorporating the components of a routine CMR study improves the determination of infarct age compared with T2-CMR alone. Journal of Cardiovascular Magnetic Resonance. BioMed Central. 2013; 15(Suppl 1): O73.

[12] Cerqueira MD, Weissman NJ, Dilsizian V, Jacobs AK, Kaul S, Laskey WK, et al. Standardized myocardial segmentation and nomenclature for tomographic imaging of the heart: a statement for healthcare professionals from the Cardiac Imaging Committee of the Council on Clinical Cardiology of the American Heart Association. Circulation. 2002 Jan 29; 105(4): 539-42.

[13] Bondarenko O, Beek AM, Hofman MB, et al. Standardizing the definition of hyperenhancement in the quantitative assessment of infarct size and myocardial viability using delayed contrast-enhanced CMR. J.Cardiovasc.Magn.Reson. 2005; 7(2): 481-5.

[14] Whalen DAJ, Hamilton DG, Ganote CE, et al. Effect of a transient period of ischemia on myocardial cells. I. Effects on cell volume regulation. Am J Pathol. 1974 Mar; 74(3): 381-97.

[15] Karolle BL, Carlson RE, Aisen AM, et al. Transmural distribution of myocardial edema by NMR relaxometry following myocardial ischemia and reperfusion. Am Heart J. 1991 Sep; $122(3 \mathrm{Pt}$ 1): 655-64.

[16] Klein HH, Nebendahl K, Schubothe M, et al. Intracoronary hyperosmotic mannitol during reperfusion does not affect infarct size in ischemic, reperfused porcine hearts. Basic Res Cardiol. 1985 May-Jun; 80(3): 251-9.

[17] Kloner RA, Rude RE, Carlson N, Maroko PR, DeBoer LW, Braunwald E. Ultrastructural evidence of microvascular damage and myocardial cell injury after coronary artery occlusion: which comes first? Circulation. 1980 Nov; 62(5): 945-52.

[18] Higgins CB, Herfkens R, Lipton MJ, et al. Nuclear magnetic resonance imaging of acute myocardial infarction in dogs: alterations in magnetic relaxation times. Am J Cardiol. 1983 Jul; 52(1): 184-8.

[19] Hsu EW, Wakatsuki Y, Aiken NR, Funabashi N, Blackband SJ, Mikami Y, et al. Nuclear magnetic resonance microscopy of single neurons under hypotonic perturbation. Am J Physiol. 1996 Dec; 271(6 Pt 1): C1895-900.

[20] Hazlewood CF, Chang DC, Nichols BL, Woessner DE. Nuclear magnetic resonance transverse relaxation times of water protons in skeletal muscle. Biophys J. 1974 Aug; 14(8): 583-606.

[21] Friedrich MG. Myocardial edema-a new clinical entity? Nat.Rev.Cardiol. 2010 Mar 23.

[22] Kuntz IDJ, Brassfield TS, Law GD, et al. Hydration of macromolecules. Science. 1969 Mar 21; 163(873): $1329-31$.

[23] Knight RA, Ordidge RJ, Helpern JA, et al. Temporal evolution of ischemic damage in rat brain measured by proton nuclear magnetic resonance imaging. Stroke. 1991 Jun; 22(6): 802-8.

[24] Deux J-F, Maatouk M, Vignaud A, Luciani A, Lenczner G, Mayer J, et al. Diffusion-weighted echo planar imaging in patients with recent myocardial infarction. Eur Radiol. 2011 Jan; 21(1): 46-53. http://dx.doi.org/10.1007/s00330-010-1912-6

[25] Kociemba A, Pyda M, Katulska K, Łanocha M, Siniawski A, Janus M, et al. Comparison of diffusion-weighted with T2-weighted imaging for detection of edema in acute myocardial infarction. J.Cardiovasc.Magn.Reson. 2013; 15: 90.

http://dx.doi.org/10.1186/1532-429X-15-90 
[26] Mann T, Goldberg S, Mudge GHJ, et al. Factors contributing to altered left ventricular diastolic properties during angina pectoris. Circulation. 1979 Jan; 59(1): 14-20. http://dx.doi.org/10.1161/01.CIR.59.1.14

[27] Bourdillon PD, Lorell BH, Mirsky I, et al. Increased regional myocardial stiffness of the left ventricle during pacing-induced angina in man. Circulation. 1983 Feb; 67(2): 316-23. http://dx.doi.org/10.1161/01.CIR.67.2.316

[28] Serizawa T, Carabello BA, Grossman W. Effect of pacing-induced ischemia on left ventricular diastolic pressure-volume relations in dogs with coronary stenoses. Circ Res. 1980 Mar; 46(3): 430-9. http://dx.doi.org/10.1161/01.RES.46.3.430

[29] Gaasch WH, Fishbein MC, Bing OH, J YR, Pine MB, Lando U, et al. Myocardial contracture during prolonged ischemic arrest and reperfusion. Am J Physiol. 1978 Dec; 235(6): H619-27.

[30] Cooke R, Wien R. The state of water in muscle tissue as determined by proton nuclear magnetic resonance. Biophys J. 1971 Dec; 11(12): 1002-17.

[31] Unger E, Littlefield J, Gado M. Water content and water structure in CT and MR signal changes: possible influence in detection of early stroke. AJNR Am J Neuroradiol. 1988 Jul-Aug; 9(4): 687-91.

[32] Ordidge RJ, Helpern JA, Knight RA, et al. Investigation of cerebral ischemia using magnetization transfer contrast (MTC) MR imaging. Magn.Reson.Imaging. 1991; 9(6): 895-902.

[33] Majno G, Joris I. Cells, Tissues and Disease: Principles of General Pathology. New York: Oxford University Press; 2004. 